\title{
Fungal Etiology of Chronic Sinusitis in Tertiary Care Hospital in Northern India
}

\author{
Sikander Chirag* and Shashi Sudhan Sharma \\ Department of Microbiology, GMC Jammu, India \\ *Corresponding author
}

Keywords

Aspergillus flavus, Chronic

rhinosinusitis, $\mathrm{KOH}$, Fungal rhinosinusitis

Article Info

Accepted:

18 December 2020

Available Online:

10 January 2021

\section{A B S T R A C T}

\section{Introduction}

Sinusitis are group of disorders leading to inflammation of mucosa of paranasal sinuses. Now termed as rhinosinusitis defined as inflammation of mucosa of nose and paranasal sinuses. The classification of rhinosinusitis includes acute rhinosinusitis (ARS) and chronic rhinosinusitis(CRS).

Acute infection may last for four weeks whwre as chronic may be more than twelve weeks. Chronic rhinosinusitis clinical diagnosis involves - presence of two or more symptoms one of which should be nasal discharge or nasal obstruction with loss of smell ${ }^{1}$.Common fungal agents causing sinusitis includes Aspergillus flavus, Mucor, Fusarium, Rhizopus etc ${ }^{2}$ Rhinosinusitis is a large financial, health and psychological burden on society ${ }^{3}$. India is common harbour for fungal mucos is due to hot and humid climate ${ }^{4}$. Diabetics, immunocompromised, cancer patients, malnourished and those on anticancer drugs are at increased risk for fungal infection ${ }^{5}$.

The aim of study was to study the profile of fungal agents among patients clinically diagnosed as chronic rhinosinusitis in GMC Jammu. 
Inclusion criteria: Patients diagnosed with chronic rhinosinusitis

Patients of all age group and both sex

Exclusion criteria: Patients with malignancy of paranasal sinuses

Patients on anti retroviral therapy

\section{Materials and Methods}

This study was conducted in GMC Jammu for a period of one year. A total of 200 samples were collected from patients of chronic rhinosinusitis attending ENT department. Specimen processing was done in the department of microbiology.

Samples collected were sinus washings, nasal mucosa, crust nasal scrapings, nasal polyp biopsy

Sample collection and transportationSpecimens were collected and transported in saline and processed as per standard procedures

\section{Laboratory diagnosis}

Direct examination-Fungal elements are directly seen in $\mathrm{KOH}$ mount preperation. For the clinical specimens a $\mathrm{KOH}$ mount was performed to identify yeast, hyphae and pseudohyphae.

Fungal culture-The clinical specimens were inoculated on Sabourauds dextrose agar with antibiotics and without cycloheximide at $25 \mathrm{c}$ and 37c.The cultures were examined daily during first week and twice a week for further four weeks before being considered as sterile.

\section{Results and Discussion}

The present study involved the mycological profile of two hundred clinically suspected cases of chronic sinusitis over a period of one year.

In the present study of 200 clinically suspected cases of chronic sinusitis, 120 were males and 80 were females as shown in table 1.

Out of 200 total cases of chronic sinusitis, 130 were from urban areas and 70 from rural areas table 2

Out of 200 cases the maximum numbers of cases were between 21-30 yrs 60 followed by 41-50 yrs 45.there was no case below 10 yrs of age and above 60 there were 5 cases table 3.

Distribution of Fungus isolates on the basis of direct and culture examination table 4

As shown in table out of 60 fungal positive specimens 18 were positive by both culture and direct $\mathrm{KOH}$ examination and 17 were $\mathrm{KOH}$ positive and culture negative.

Distribution of Fungal isolates in clinical cases of chronic rhinosinusitis table 5 .

The most common fungal isolate was Aspergillus flavus followed by Mucor. Aspergillus fumigatus and rhizopus were next commonly isolated.

Chronic sinusitis is a significant health care problem and results in large financial burden on society. It is a common disorder affecting approximately $20 \%$ of population ${ }^{4}$. Due to increased awareness and latest technology paranasal mycosis has been recognized more frequently in different parts of world. Multi drug resistant sinusitis and no response to antibiotics leads us to consider mycosis as cause of sinusitis. The present study was undertaken to study the mycological profile of 200 cases of chronic sinusitis over a period of one year in GMC Jammu. 
Table.1

\begin{tabular}{|l|l|l|}
\hline Total Number Of Cases & Male & Female \\
\hline 200 & 120 & 80 \\
\hline
\end{tabular}

Table.2

\begin{tabular}{|l|l|l|}
\hline $\begin{array}{l}\text { Total number Of } \\
\text { Cases }\end{array}$ & Rural & Urban \\
\hline $\mathbf{2 0 0}$ & 70 & 130 \\
\hline
\end{tabular}

Table.3

\begin{tabular}{|l|c|}
\hline Age In Years & Number of Patients Tested \\
\hline $\mathbf{0 - 1 0}$ & Nil \\
\hline $\mathbf{1 1 - 2 0}$ & 20 \\
\hline $\mathbf{2 1 - 3 0}$ & 60 \\
\hline $\mathbf{3 1 - 4 0}$ & 40 \\
\hline $\mathbf{4 1 - 5 0}$ & 45 \\
\hline $\mathbf{5 1 - 6 0}$ & 30 \\
\hline Above 60 & 5 \\
\hline
\end{tabular}

Table.4

\begin{tabular}{|l|l|l|}
\hline KOH Results & Culture Positive On SDA & Culture Negative on SDA \\
\hline Positive 35 & 18 & 17 \\
\hline Negative 165 & 25 & 140 \\
\hline
\end{tabular}

Table.5

\begin{tabular}{|l|l|}
\hline Type of Fungus & No of Cases \\
\hline Aspergillus flavus & 20 \\
\hline Aspergillus fumigatus & 3 \\
\hline Cladophialophora & 2 \\
\hline Alternaria & 2 \\
\hline Rhizopus & 3 \\
\hline Fusarium & 1 \\
\hline Mucor & 6 \\
\hline Exophiala & 1 \\
\hline Acremonium & 1 \\
\hline Candida albicans & 2 \\
\hline Non candida albicans & 2 \\
\hline
\end{tabular}

In our study there was predominance of chronic sinusitis in male patients which is concordant with other studies 6,7 . This may be due to males are most commonly exposed to irritating pollutants to factories and traffic. 
In our study again more patients were from urban areas $^{7}$ due to most possible exposure to pollution and dust. Maximum number of patients were in age group 21-30 yrs as observed by others ${ }^{8}$.

In this study fungal positivity by both culture and koh examination was 60 .Out of these 18 were positive by both culture and $\mathrm{KOH}$ examination and 17 were $\mathrm{KOH}$ positive and culture negative which could be due any faulty technique, antifungal therapy etc. The most common isolate was Aspergillus flavus and mucor. This could be due to dust containing these fungal agents and also they are commonly found in environment. Other fungal isolates were aspergillus fumigatus, Cladophialophora, Alternaria, Rhizopus, Fusarium, Exophiala, Acremonium, candida non albicans and candida albicans which was shown by other studies ${ }^{9,10}$. This may be due to hot and humid climate in this geographical area. Fungal agents like Acremonium, fusarium, Exophiala, etc as rare but emerging as new fungal etiological agents in chronic sinusitis. They need to be managed effectively for treating chronic sinusitis as treatment cost is more and they may not respond well to lot of antifungal agents.

In conclusion, this study highlights the importance of paranasal sinus mycosis in North India. As fungal diseases are not notifiable infections like viral, bacterial or parasitic disease hence these are not given much attention and usually diagnosis ids established very late. Therefore early diagnosis and recognition of fungal sinusitis is very important, not only because it is curable in the early stages, but also to prevent progression of disease to more destructive and serious forms. These days due to advanced techniques and increase in awareness of people there is better recognition of this disease entity. Our suggestion is that all rhinosinusitis cases should be screened for fungal aetiology.

\section{References}

1. Fokkens WJ,Lund VJ,Mulloj J,et al.Epos 2012:European position paper on rhinosinusitis and nasal polyps 2012.Rhinology.2012;50(suppl 23):1298.

2. Gleeson M,browning G,Burton $M$ et al.Fungal sinusitis Scott Brown otorhinolaryngol head and Neck Surg 2008;7:1440-52

3. Ray NF,Baraniuk JN et al.Health care expenditures for sinusitis in 1996:Contributions of asthma,rhinitis and other airway disorders; $J$ allergyClin Immunol 1993;103:408-14

4. Chakrabati A,Sharma SC.Paranasal sinus mycoses.Ind $\mathbf{J}$ Chest Dis Allied Sci 2000;293-304.

5. Hutter RVP et al.Diagnosis and management of cancer.Ann Otol Laryngol 1973;77:29

6. Prateek S,Banerjee G,Gupta P,Singh M et al.fungal rhinosinusitis:A prospective study in university hospital of Uttar pradesh.Indian journal of medical Microbiology 2013;31(3):266-9.

7. Shone GR.Bacteriology of chronic maxillary sinusitis.J Laryngol and Otol 1973;101.

8. Merica FW.Ann Otol laryngol Rhin and Laryngol.1938;48:1011.

9. Michael RC,Michael JS,Ashbee RH et al., Mycological profile of fungal sinusitis:An audit of specimens over 7 year period in a tertiary care hospital in tamilnadu.Indian J Patol Microbiol.2008;51(4):493-6

10. Veress et al:Observation on primary paranasal aspergillus granuloma in Sudan.Am J Tropical Med Hyg 1973;22:765-72. 
How to cite this article:

Sikander Chirag and Shashi sudhan sharma. 2021. Fungal Etiology of Chronic Sinusitis in Tertiary Care Hospital in Northern India. Int.J.Curr.Microbiol.App.Sci. 10(01): 2999-3003. doi: https://doi.org/10.20546/ijcmas.2021.1001.347 